\title{
Intelligent multi-objective model predictive control applied to steam turbine start-up
}

\author{
Masakazu SHIRAKAWA*, Yeboon YUN** and Masao ARAKAWA*** \\ *Toshiba Energy Systems \& Solutions Corporation \\ 2-4 Suehiro-cho, Tsurumi-ku, Yokohama-shi, Kanagawa 230-0045, Japan \\ E-mail: masakazu1.shirakawa@toshiba.co.jp \\ **Faculty of Environmental and Urban Engineering, Kansai University \\ 3-3-35 Yamate-cho, Suita-shi, Osaka 564-8680, Japan \\ ${ }^{\star * \star}$ Department of Reliability-based Information System Engineering, Kagawa University \\ 2217-20 Hayashi-cho, Takamatsu-shi, Kagawa 761-0396, Japan
}

Received: 28 August 2017; Revised: 24 November 2017; Accepted: 19 December 2017

\begin{abstract}
This study proposes an intelligent multi-objective model predictive control method in which an artificial neural network and a genetic algorithm are used to realize satisficing decision-making, which is an interactive multi-objective programming technique. We considered model predictive optimization under a dynamic environment with multiple objectives. To predict nonlinear function forms with dynamic plant characteristics, we applied a recurrent radial basis function network, which is a type of artificial neural network. For optimization with multiple objectives, we applied a satisficing trade-off method along with metaheuristic optimization in the form of genetic algorithms. The features of this control method are as follows. (1) Several conflicting control objectives can be optimized in online control based on multi-objective evaluation through human-computer interaction and (2) an optimal and flexible plant control can be performed within a restrained practical computing time for real-time applications, with acceptable control quality using online adaptive model prediction. This study demonstrates the success of model prediction using computational intelligence combined with an interactive optimization technique for multi-objective model predictive control problems by applying the proposed method to steam turbine start-up control with multiple objectives consisting of the start-up time and rotor thermal stress of the steam turbine. The dynamic simulation results showed an effective control performance within a reasonable computing time.
\end{abstract}

Keywords : Genetic algorithm, Model predictive control, Multi-objective optimization, Power plant, Radial basis function network, Recurrent neural network, Satisficing trade-off method, Steam turbine

\section{Introduction}

When function forms in mathematical models cannot be given explicitly in terms of design variables, the function values are usually determined by performing numerical or real experiments. Since these experiments are often expensive, it is important to develop techniques for obtaining solutions with as few experiments as possible. To this end, model predictive optimization is intended to find optimal solutions in parallel with predicting the function forms in mathematical models. Metamodeling (Wang and Shan, 2006) and surrogate-based optimization (also called sequential approximate optimization; Nakayama et al., 2009) methods apply the same concepts. Several methods have been developed for this purpose, of which the response surface (Myers and Montgomery, 2016), design of experiments (Montgomery, 2017), Kriging (Cressie, 1988), artificial neural network (Papadrakakis et al., 1998), and support vector machine (Yun and Nakayama, 2016) methods are well known but mainly handle static optimization. For dynamic optimization problems, the model predictive control method (Maciejowski, 2002) has been developed using ideas analogous to those mentioned above. However, the objectives of almost all conventional model predictive control studies have been to optimize a single-objective function (e.g., only time-optimal control problem). Research on 
multiple-objective control problems has been restricted in its applications (Bempord and Muñoz de la Peña, 2009; Zavala and Flores-Tlacuahuac, 2012; Núñez et al., 2014). In cases with multi-objective functions, it is very difficult to adjust the weights of the objective functions using a trial-and-error heuristic approach. Moreover, the computing time increases drastically upon generation of a Pareto frontier in these cases, making practical control problems impossible. To avoid these assignments, it is necessary to determine a priority beforehand among multiple objectives (Rojas et al., 2013; He et al., 2015).

To balance computing time and accuracy, Shirakawa et al. (2007) proposed a new intelligent optimization algorithm that combines surrogate-based optimization and the satisficing trade-off method (Nakayama, 1995), which is an interactive multi-objective programming technique. Furthermore, the authors (Shirakawa et al., 2007) reported that they developed a multi-objective optimization system for thermal power plant operations wherein an operator can interact with a computer to optimize a start-up schedule based on multi-objective evaluation. However, since this system deals with a dynamic optimization problem by transposing it to a static optimization problem, it must initially determine the entire start-up schedule offline. That is, although this system can optimize multi-objective functions, it cannot be applied to online optimal control problems. Such systems cannot cope with unexpected changes in thermodynamic state values resulting from disturbances during plant operations. Several online optimal control methods for steam turbine start-up using model predictive control have been proposed (Nakai et al., 1996; D'Amato, 2006; Biyik et al., 2012; Tica et al., 2012; Schuermann et al., 2016); however, the objectives of these studies have been to optimize only a single-objective time-optimal control function. Typically, steam turbine start-up control for a thermal power plant involves several conflicting objective functions, such as those for faster start-up, less thermal stress due to reduced lifetime consumption of the steam turbine rotors, reduced fuel consumption, and reduced auxiliary power consumption. Given recent developments in electricity deregulation and increases in renewable energy, there is a greater need for thermal power plant operations to become more flexible and able to respond to market changes.

Therefore, the authors extended the previously reported optimization algorithm (Shirakawa et al., 2007) for online optimal control. In this paper, for a dynamic environment with multiple objectives, we propose a multi-objective model predictive control method using computational intelligence, which is specifically a recurrent radial basis function network (RRBFN) and satisficing trade-off method. This method maintains the framework of previously reported human-computer interaction features and can be applied to online optimal control from among multiple objectives within a reasonable computing time. The proposed method is applied herein to dynamic simulations of a $600 \mathrm{MW}$ steam power plant, and its effectiveness is demonstrated using the example of steam turbine start-up control with multiple objectives consisting of the start-up time and rotor thermal stress of the steam turbine.

\section{Proposed multi-objective model predictive control}

Consider a dynamic optimization problem. Following the optimal control theory, let $u$ denote the control input and $\boldsymbol{x}=\left[x_{1}, x_{2}, \cdots, x_{n}\right]^{\mathrm{T}}$ denote the vector of state variables. The theory assumes the following mathematical model:

$$
\begin{aligned}
& \min _{\boldsymbol{x}, u} f=\varphi[\boldsymbol{x}(T)]+\sum_{k=0}^{T-t-1} V[\boldsymbol{x}(t+k), u(t+k), t+k], \\
& \text { subject to }\left\{\begin{array}{l}
\boldsymbol{x}(t+k+1)=v[\boldsymbol{x}(t+k), u(t+k), t+k], \quad \boldsymbol{x}(t)=\boldsymbol{x}_{o}, \\
\boldsymbol{g}[\boldsymbol{x}(t+k), u(t+k), t+k] \leq 0, \quad k=0,1, \cdots, T-t-1,
\end{array}\right.
\end{aligned}
$$

where $\boldsymbol{g}=\left[g_{1}, g_{2}, \cdots, g_{s}\right]^{\mathrm{T}}$ represents the vector of the constraining conditions; $t$, the present time; $T$, the terminal time $(T \geq t)$; and $\boldsymbol{x}_{o}$, the vector value of actual observed state variables at time $t$.

If the function form in the above model is given explicitly, then some techniques from optimal control theory can be applied. However, we assume that some function forms cannot explicitly be given due to their nonlinear and complex models. In this case, we predict some future states $\boldsymbol{x}(t+1), \boldsymbol{x}(t+2), \cdots, \boldsymbol{x}\left(t+p_{1}\right)$ for the control input sequence $u(t+1), u(t+2), \cdots, u\left(t+p_{2}\right)$, where $p_{1} \geq p_{2}, p_{1}$ represents the prediction period, and $p_{2}$ represents the control period. Our aim is to determine the optimal control input sequence $u(t+k)$ over $[t, T]$.

Suppose that the problem has the following multiple objectives:

$$
\boldsymbol{f}=\left[f_{1}, f_{2}, \cdots, f_{r}\right]^{\mathrm{T}}
$$


For example, in the case of a thermal power plant, those objectives are the start-up time, lifetime consumption rate, fuel consumption rate, and pollutant emission rate of the machine. To obtain a final decision for those multi-objective problems, we apply the satisficing trade-off method (Nakayama, 1995) which is an aspiration-level-based method (to be described in Section 3.1). For predicting the future states, we apply an artificial neural network technique, namely, an RRBFN (to be described in Section 3.3.1). The original radial basis function network (RBFN) provides less training computing time than other artificial neural networks. Further, the optimal control input sequence closest to the aspiration level is searched in conjunction with the RRBFN prediction model using a genetic algorithm (GA) (which we describe in Section 3.2).

Figure 1 illustrates the structure of the multi-objective model predictive control system developed in this study. This system applies an interactive multi-objective programming technique called the satisficing trade-off method as its framework via human-computer interaction and integrates the intelligence of the computer with the knowledge of the user. Figure 2 shows an example of the discrete model predictive control scheme. Referring to Figs. 1 and 2, we explain the procedure of the proposed method with discrete time periods.

Step 1. Controller: The RRBFN prediction model is trained to predict the dynamic system model based on observed past states $\boldsymbol{x}$ and control input sequence $u$. The training datasets for the RRBFN prediction model are as follows:

$$
\left\{\begin{array}{l}
\boldsymbol{x}\left(k-p_{3}\right), \boldsymbol{x}\left(k-p_{3}+1\right), \cdots, \boldsymbol{x}(k), \\
u\left(k-p_{3}\right), u\left(k-p_{3}+1\right), \cdots, u(k), \quad k=t-p+1, t-p+2, \cdots, t,
\end{array}\right.
$$

where $p$ denotes the depth of the sampling training data, and $p_{3}$ represents the past sample period.

Step 2. Human interface: The user sets the aspiration level (desired values) $\bar{f}$ corresponding to each objective function as follows:

$$
\overline{\boldsymbol{f}}=\left[\overline{f_{1}}, \overline{f_{2}}, \cdots, \overline{f_{r}}\right]^{\mathrm{T}}
$$

Step 3. Controller: The optimal solution for control input rate sequence $\Delta u^{\text {best }}$ (or control input sequence $u^{\text {best }}$ ) closest to $\bar{f}$ is sought in conjunction with the RRBFN prediction model using a GA:

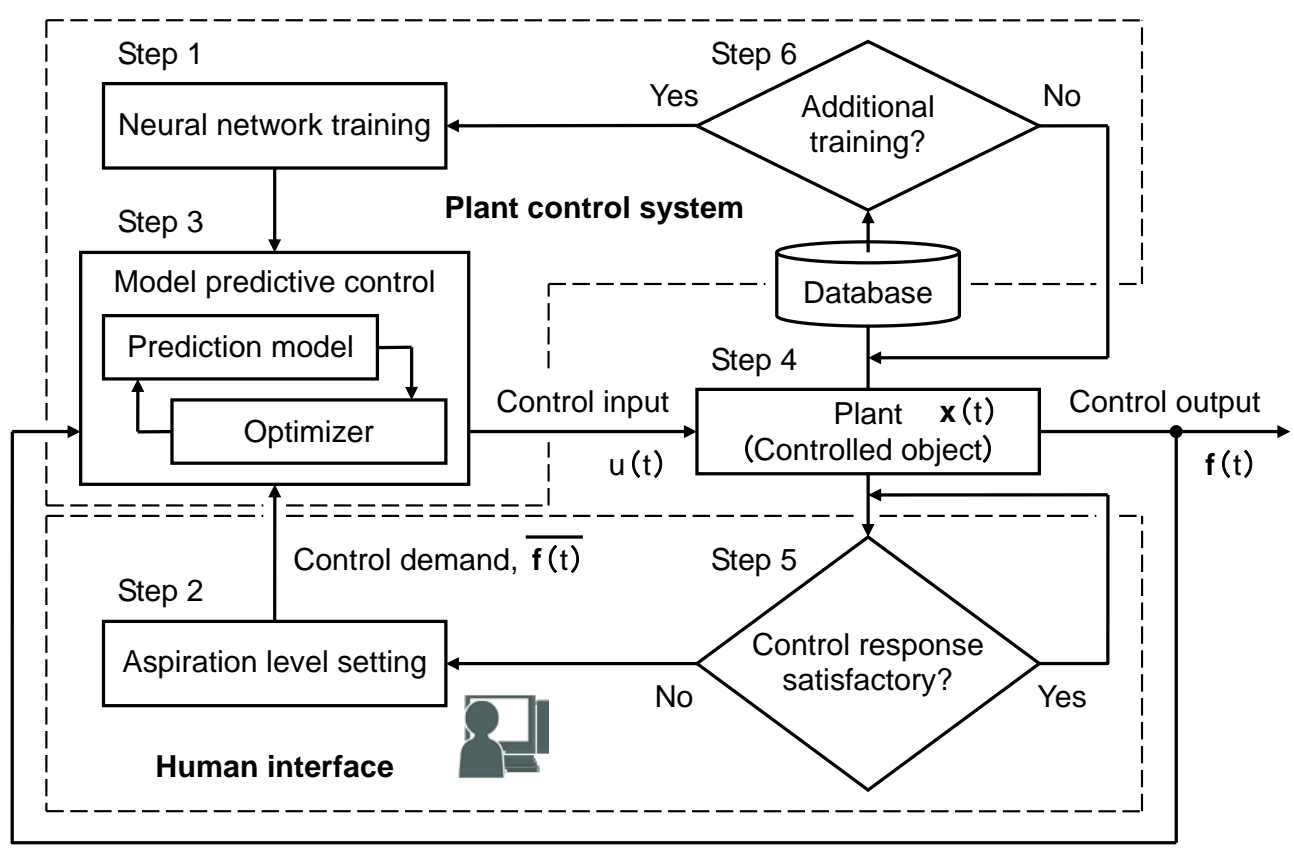

Fig. 1 Structure of the proposed multi-objective model predictive control system. A cooperative humanmachine system is proposed to optimize control input $u(t)$ by online control to aspiration level $\overline{\boldsymbol{f}(t)}$ from among multiple objectives $\boldsymbol{f}(t)$ at each sampling time $t$. The training datasets based on observed stats $\boldsymbol{x}(t)$ for the RRBFN prediction model are stored in the database. 


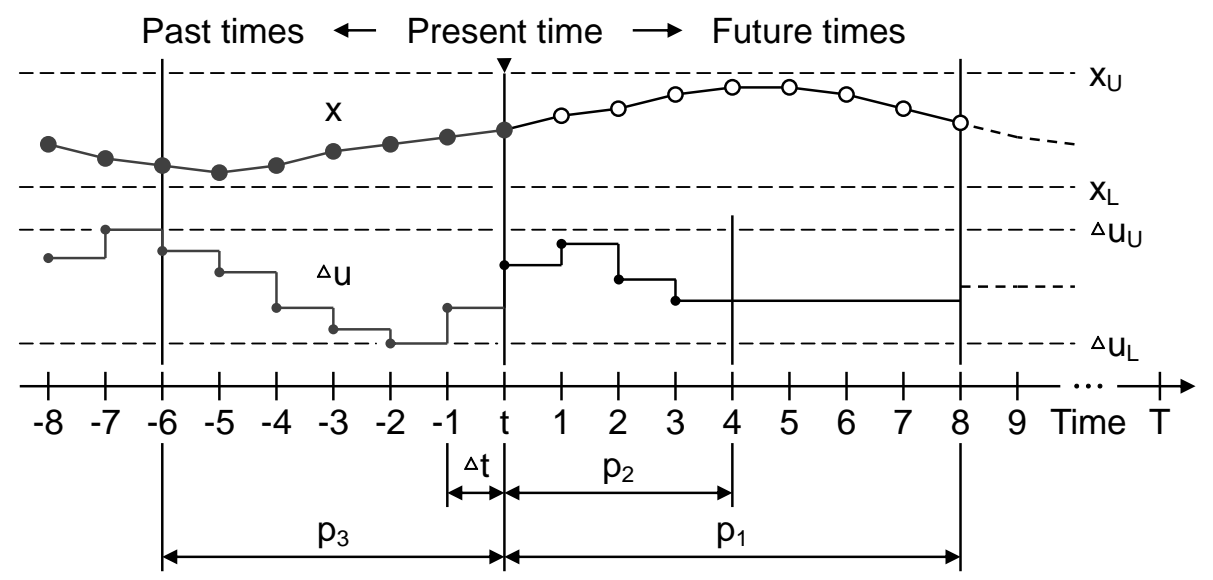

Fig. 2 Discrete model predictive control scheme. This figure is an example, with a prediction period $p_{1}$ of 8 samples, control period $p_{2}$ of 4 samples, and past sample period $p_{3}$ of 6 samples, where $t$ represents the present time; $T$, the terminal time; $\Delta t$, one sampling period; $\Delta u_{L}$ and $\Delta u_{U}$, the lower and upper bounds, respectively, for control input rate $\Delta u$; and $x_{L}$ and $x_{U}$, the lower and upper bounds, respectively, for state $x$. These parameters are adjusted based on control problems.

i. $\quad N$ initial individuals of control input rate sequence $\Delta u_{j}$ are generated randomly as follows:

$\Delta u_{L 1} \leq \Delta u_{j}(t+k) \leq \Delta u_{U 1}, \quad j=1,2, \cdots, N, \quad k=0,1, \cdots, p_{2}-1$,

with $\Delta u_{j}(t+k)=\Delta u_{L 2} \leq \Delta u_{j}\left(t+p_{2}-1\right) \leq \Delta u_{U 2}, \quad j=1,2, \cdots, N, \quad k=p_{2}, p_{2}+1, \cdots, p_{1}-1$,

where $\Delta u_{L 1}$ and $\Delta u_{U 1}$ represent the lower and upper bounds, respectively, during control period $p_{2}$; and $\Delta u_{L 2}$ and $\Delta u_{U 2}$, the lower and upper bounds, respectively, during prediction period $p_{1}$ beyond $p_{2}$.

ii. The future state values of dynamic system model $\boldsymbol{x}_{j}$ for each control input sequence $u_{j}$ are predicted using the RRBFN model during prediction period $p_{1}$ :

$$
\left\{\begin{array}{l}
\boldsymbol{x}_{j}(t+k+1)=\hat{v}\left[\boldsymbol{x}_{j}(t+k), u_{j}(t+k), t+k\right], \\
u_{j}(t+k+1)=u_{j}(t+k)+\Delta u_{j}(t+k), \quad j=1,2, \cdots, N, \quad k=0,1, \cdots, p_{1}-1,
\end{array}\right.
$$

where $\hat{v}$ denotes the predicted function of dynamic system model $v$ in Eq. (1), and $u_{j}(t)$ and $\boldsymbol{x}_{j}(t)$ are set to actual observed values $u_{j}(t)=u_{o}$ and $\boldsymbol{x}_{j}(t)=\boldsymbol{x}_{o}$, respectively.

iii. For future state values of dynamic system model $\boldsymbol{x}_{j}$, for each control input sequence $u_{j}$, the value of the auxiliary scalar function of the satisficing trade-off method is estimated for each $F_{j}$ :

$$
F_{j}=\max _{1 \leq i \leq r} \frac{f_{i}\left(\boldsymbol{x}_{j}, u_{j}\right)-\overline{f_{i}}}{\overline{f_{i}}-f_{i}^{*}}+\alpha \sum_{i=1}^{r} \frac{f_{i}\left(\boldsymbol{x}_{j}, u_{j}\right)}{\overline{f_{i}}-f_{i}^{*}}+\sum_{l=1}^{s} \beta_{l} \max _{l}\left[0, g_{l}\left(\boldsymbol{x}_{j}, u_{j}\right)\right]^{2}, \quad j=1,2, \cdots, N,
$$

where $f_{i}^{*}$ is an ideal value of the $i$-th objective function, and $u_{j}$ and $\boldsymbol{x}_{j}$ are $u_{j}=u_{j}(t+1), u_{j}(t+2), \cdots$, $u_{j}\left(t+p_{1}\right)$ and $\boldsymbol{x}_{j}=\boldsymbol{x}_{j}(t+1), \boldsymbol{x}_{j}(t+2), \cdots, \boldsymbol{x}_{j}\left(t+p_{1}\right)$, respectively. The third term on the right side of Eq. (8) is the penalty function, where penalty parameter $\beta_{l}$ is a sufficiently large positive number for each constraining condition $g_{l}$.

iv. The individuals in control input rate sequence $\Delta u_{j}$ are evaluated using the value of $F_{j}$, and $N$ new individuals of $\Delta u_{j}$ are generated through natural selection and genetic operators.

v. Steps ii-v are repeated until a stop condition - for example, the number of iterations - occurs.

vi. The best control input rate sequence $\Delta u^{\text {best }}$ that minimizes the value of $F_{j}$ is decided.

Step 4. Plant: Only the best control input rate $\Delta u^{\text {best }}(t)$ at time $t$ is extracted, and the control input $u(t+1)$ $=u(t)+\Delta u^{\text {best }}(t)=u_{o}+\Delta u^{\text {best }}(t)$ is input to the plant. Actual state values $\boldsymbol{x}(t+1)$ according to $u(t+1)$ are 
observed, and $u_{o}$ and $\boldsymbol{x}_{o}$ are set to actual observed values $u_{o}=u(t+1)$ and $\boldsymbol{x}_{o}=\boldsymbol{x}(t+1)$, respectively. Stop if $t+1=T$; otherwise, perform the update $t \leftarrow t+1$, and return to Step 3. However, if the user wants to modify the aspiration level, go to Step 5, and/or if the RRBFN predictive model requires additional training, go to Step 6 .

Step 5. Human interface: The user judges whether the observed control responses are satisfactory. If the user is not satisfied, the user modifies the aspiration level. Subsequently, Steps 2-5 are repeated until the user obtains agreeable control responses. If the user is satisfied, return to the end of Step 4.

Step 6. Controller: If approximation errors between actual observed values and predicted values are large, the RRBFN prediction model is further trained (offline or online). Subsequently, Steps 1-6 are repeated until the approximation errors are reduced. If the approximation errors are small, return to the end of Step 4.

\section{Intelligent optimization method}

In this section, we detail a new intelligent optimization method for multi-objective model predictive control. This method combines an RRBFN with a GA and employs the satisficing trade-off method to enable multi-objective optimization of plant controls. The reason that we focus on the satisficing trade-off method is that it does not require the generation of a Pareto frontier or priorities and weight adjustments for multiple objectives, as was necessary in previous multi-objective control studies. Furthermore, in model predictive control, technology for predicting dynamic plant characteristics is important. To achieve real-time control performance, we devised a recurrent neural network that extends existing RBFNs and integrates an efficient data degeneracy method using cluster analysis to shorten its learning time. In addition, the accuracy of this prediction model was verified using actual thermal power plant operation data.

\subsection{Satisficing trade-off method}

Multi-objective optimization implies the simultaneous optimization of multiple objective functions that have a trade-off relationship with one another under a given condition. This problem is typically expressed as follows:

$$
\left\{\begin{array}{l}
\min _{\boldsymbol{x}} \boldsymbol{f}(\boldsymbol{x})=\left[f_{1}(\boldsymbol{x}), f_{2}(\boldsymbol{x}), \cdots, f_{r}(\boldsymbol{x})\right]^{\mathrm{T}}, \\
\text { subject to } g_{l}(\boldsymbol{x}) \leq 0, \quad l=1,2, \cdots, s,
\end{array}\right.
$$

where $\boldsymbol{x}=\left[x_{1}, x_{2}, \cdots, x_{n}\right]^{\mathrm{T}}$ is a vector of design variables, $f$ represents the objective functions, and $g$ specifies the constraining conditions (operational constraints).

Because it is difficult to find the solution if the problem is expressed in such a vector format, the problem is scalarized for ease of solving. In this case, when the formula is scalarized by using the satisficing trade-off method, which is an interactive multi-objective programming technique employing the aspiration-level-based method. See (Shirakawa et al., 2007) for more details. The following auxiliary scalar optimization of Eq. (11) is obtained, using the Tchebyshev scalarization function $F$ of Eq. (10). Here, the operational constraints can be included in Eq. (11) as the penalty function.

$$
\begin{aligned}
& F(\boldsymbol{x})=\max _{1 \leq i \leq r} w_{i}\left[f_{i}(\boldsymbol{x})-\overline{f_{i}}\right]+\alpha \sum_{i=1}^{r} w_{i} f_{i}(\boldsymbol{x}), \\
& \min _{\boldsymbol{x}} F(\boldsymbol{x})+\sum_{l=1}^{s} \beta_{l} \max _{l}\left[0, g_{l}(\boldsymbol{x})\right]^{2},
\end{aligned}
$$

where $\alpha$ is usually set to a sufficiently small positive number (such as $10^{-6}$ ), and the penalty parameter $\beta_{l}$ is a sufficiently large positive number for each constraining condition $g_{l}$. Weight $w_{i}$ can be expressed as follows:

$$
w_{i}=\frac{1}{f_{i}-f_{i}^{*}},
$$

where $\overline{f_{i}}$ is the aspiration level (desired values) of the objective functions, and $f_{i}^{*}$ is an ideal point, usually given 
such that $f_{i}^{*} \leq \min \left[f_{i}(\boldsymbol{x}) \mid \boldsymbol{x} \in \boldsymbol{X}\right]$, where $\boldsymbol{X}$ is the set of all feasible solutions.

\subsection{GA with blend alpha crossover}

Since we focused on continuous-variable optimization, Eq. (11) was minimized using a real-coded GA. Crossover is the most important operator in real-coded GAs. We discuss the application of a blend alpha crossover (BLX-a) (Eshelman and Schaffer, 1993) in this paper. BLX-a combines two parents $P^{1}$ and $P^{2}$ to generate two offspring $C^{1}$ and $C^{2}$ with a crossover probability by sampling new values in the range $\left[\min _{i}-I \cdot a, \max _{i}+I \cdot a\right]$ at each gene $i$. Here, $\min _{i}$ and $\max _{i}$ are the smallest and largest values, respectively, of the two parents at each gene $i$, and $I=\max _{i}-\min _{i} . a$ is usually set to 0.5 . The new offspring can be adjusted as necessary to fit within the following bounds:

$$
C_{i}^{b}=\left(\min _{i}-I \cdot a\right)+\gamma^{b} \cdot\left|\left(\max _{i}+I \cdot a\right)-\left(\min _{i}-I \cdot a\right)\right|, \quad b=1,2,
$$

where $\gamma^{b}$ is a random number between 0 and 1 .

The numerical algorithm for GA with BLX-a that is discussed in this paper has the following steps:

Step 1. Initialize: Randomly generate $N$ individuals of the initial parent generation.

Step 2. Crossover: Generate offspring using BLX-a.

Step 3. Evaluation: The fitness function value is in the Tchebyshev scalarization function $F$ of Eq. (10). The fitness function values of each individual are calculated with the penalty function of the constraining conditions, as in Eq. (11). Step 4. Selection: When selecting the fittest individuals to pass to the next generation, elite retention selection is used. That is to say, $N$ elite individuals with small fitness function values survive in the next generation. Further, Steps $2-4$ are repeated until the maximum number of iterations or maximum computing time is reached. Here, we define the maximum computing time to be within one sampling period (optimization cycle) $\Delta t$, as shown in Fig. 2.

\subsection{Model prediction using an RBFN}

An RBFN is a type of neural network that consists of several radial basis functions (Nakayama et al., 2002) and contains three layers: input, hidden, and output layers. The input values are each assigned to a neuron in the input layer and passed directly to the hidden layer without weights. A Gaussian function is used as an activation function for the hidden neurons. Subsequently, linear output weights are added between the hidden and output layers. The overall input-output mapping equation of an RBFN is presented as follows:

$$
\begin{aligned}
& O(\boldsymbol{x})=\sum_{j=1}^{q} w_{j} h_{j}(\boldsymbol{x}), \\
& \text { where } h_{j}(\boldsymbol{x})=\exp \left(\frac{-\left\|\boldsymbol{x}-\boldsymbol{c}_{j}\right\|^{2}}{R_{j}^{2}}\right)
\end{aligned}
$$

and $c_{j}$ is the center of the hidden neurons; $h_{j}$, the Gaussian function; $O$, the output values; $q$, the number of hidden neurons; $R_{j}$, the radial parameter; $w_{j}$, the weights; $\boldsymbol{x}=\left[x_{1}, x_{2}, \cdots, x_{n}\right]^{\mathrm{T}}$, the vector of input values; and $\|\cdot\|$, the Euclidean norm.

The training equation of an RBFN is defined as follows:

$$
\min _{\boldsymbol{w}} E(\boldsymbol{w})=\sum_{i=1}^{p}\left[y_{i}-O\left(\boldsymbol{x}_{i}\right)\right]^{2}+\sum_{j=1}^{q} \lambda_{j} w_{j}^{2}
$$

where $p$ is the number of training values; $\boldsymbol{w}=\left[w_{1}, w_{2}, \cdots, w_{q}\right]^{\mathrm{T}}$, the weight vector; and $y_{i}$, the training values. $\lambda_{j}$ is introduced for regularization (in this case, it was set to $10^{-7}$ ). Equation (16) can be reduced to the normal equation as follows:

$$
\boldsymbol{H}^{\mathrm{T}} \boldsymbol{y}=\boldsymbol{H}^{\mathrm{T}} \boldsymbol{O}+\lambda \boldsymbol{I} \boldsymbol{w}=\boldsymbol{H}^{\mathrm{T}} \boldsymbol{H} \boldsymbol{w}+\lambda \boldsymbol{I} \boldsymbol{w}=\left(\boldsymbol{H}^{\mathrm{T}} \boldsymbol{H}+\lambda \boldsymbol{I}\right) \boldsymbol{w}
$$


where

$$
\begin{aligned}
& \boldsymbol{H}=\left[\begin{array}{cccc}
h_{1}\left(\boldsymbol{x}_{1}\right) & h_{2}\left(\boldsymbol{x}_{1}\right) & \cdots & h_{q}\left(\boldsymbol{x}_{1}\right) \\
h_{1}\left(\boldsymbol{x}_{2}\right) & h_{2}\left(\boldsymbol{x}_{2}\right) & \cdots & h_{q}\left(\boldsymbol{x}_{2}\right) \\
\vdots & \vdots & \ddots & \vdots \\
h_{1}\left(\boldsymbol{x}_{p}\right) & h_{2}\left(\boldsymbol{x}_{p}\right) & \cdots & h_{q}\left(\boldsymbol{x}_{p}\right)
\end{array}\right], \boldsymbol{y}=\left[\begin{array}{c}
y_{1} \\
y_{2} \\
\vdots \\
y_{p}
\end{array}\right], \boldsymbol{O}=\left[\begin{array}{c}
O\left(x_{1}\right) \\
O\left(x_{2}\right) \\
\vdots \\
O\left(x_{p}\right)
\end{array}\right], \lambda \boldsymbol{I}=\left[\begin{array}{cccc}
\lambda_{1} & 0 & \cdots & 0 \\
0 & \lambda_{2} & \cdots & 0 \\
\vdots & \vdots & \ddots & \vdots \\
0 & 0 & \cdots & \lambda_{q}
\end{array}\right], \boldsymbol{w}=\left[\begin{array}{c}
w_{1} \\
w_{2} \\
\vdots \\
w_{q}
\end{array}\right], \\
& \because \quad \boldsymbol{w}=\left(\boldsymbol{H}^{\mathrm{T}} \boldsymbol{H}+\lambda \boldsymbol{I}\right)^{-1} \boldsymbol{H}^{\mathrm{T}} \boldsymbol{y}=\boldsymbol{A}^{-1} \boldsymbol{H}^{\mathrm{T}} \boldsymbol{y} .
\end{aligned}
$$

Hence, RBFN training can be solved directly with the linear simultaneous equation in Eq. (19). As a result, the training speed improves significantly and local minima are avoided. Moreover, even if the RBFN is not relearned from the beginning by updating the inverse matrix $\boldsymbol{A}^{-1}$, additional RBFN learning and forgetting can be performed easily,

$$
\text { where } \boldsymbol{A}^{-1}=\left(\boldsymbol{H}^{\mathrm{T}} \boldsymbol{H}+\lambda \boldsymbol{I}\right)^{-1} .
$$

Our proposed method generates approximate functions by combining the polynomial functions and RBFN as follows:

$$
O^{\prime}(x)=\zeta(x)+O(x)
$$

where $O$ represents the RBFN output values from Eq. (14); $O^{\prime}$, the approximate functions; and $\zeta$, the polynomial functions. The polynomial functions are used as base functions, and the RBFN estimates errors from them. This method can improve the accuracy near the side constraints of the design variables, where the training values become sparse.

\subsubsection{Proposed RRBFN prediction model}

We propose an RRBFN, shown in Fig. 3, which was applied to predict the dynamic plant characteristics in this study. The output values are predictive values at time $t+1$ :

$$
O_{1}(k), O_{2}(k), \cdots, O_{n_{3}}(k), \quad k=t+1
$$

The input values are divided into the following three types:

1. time-dependent dynamic state values $x d$ from time $t-p_{3}+1$ to $t+1$,

2. less/non-time-dependent static state values $x s$ at time $t+1$, and

3. time-dependent feedback predictive values (output values) $O$ from time $t-p_{3}+1$ to $t$.

$$
\left\{\begin{array}{l}
x d_{1}(k), x d_{2}(k), \cdots, x d_{n_{1}}(k), \quad k=t-p_{3}+1, \cdots, t, t+1, \\
x s_{1}(k), x s_{2}(k), \cdots, x s_{n_{2}}(k), \quad k=t+1 \\
O_{1}(k), O_{2}(k), \cdots, O_{n_{3}}(k), \quad k=t-p_{3}+1, \cdots, t-1, t
\end{array}\right.
$$

where $p_{3}$ is the past sampling period, and $n_{1}$ represents the number of dynamic state values; $n_{2}$, the number of static state values; $n_{3}$, the number of output values; $O$, the output values; $t$, the present time; $x d$, the dynamic state values; and $x s$, the static state values. Hence, the total number of input values is $n=n_{1} \times\left(p_{3}+1\right)+n_{2}+n_{3} \times p_{3}$. The RRBFN training method is similar to that of RBFNs, as described above, although the training values are given by the set of time-series data.

\subsubsection{Cluster analysis for RRBFN training data}

Many RRBFN training values can be obtained using a plant supervisory control and data acquisition (SCADA) system. However, because detailed sampling of long large-scale time-series data is used, RRBFN training is extremely time-consuming. The computation time depends on the size of the training data. To stay below a fixed computing time, we thin out the stored plant operation data on the SCADA system using cluster analysis before the RRBFN is trained. The cluster analysis procedure is designed to group observations based on similarities between them. The observations 


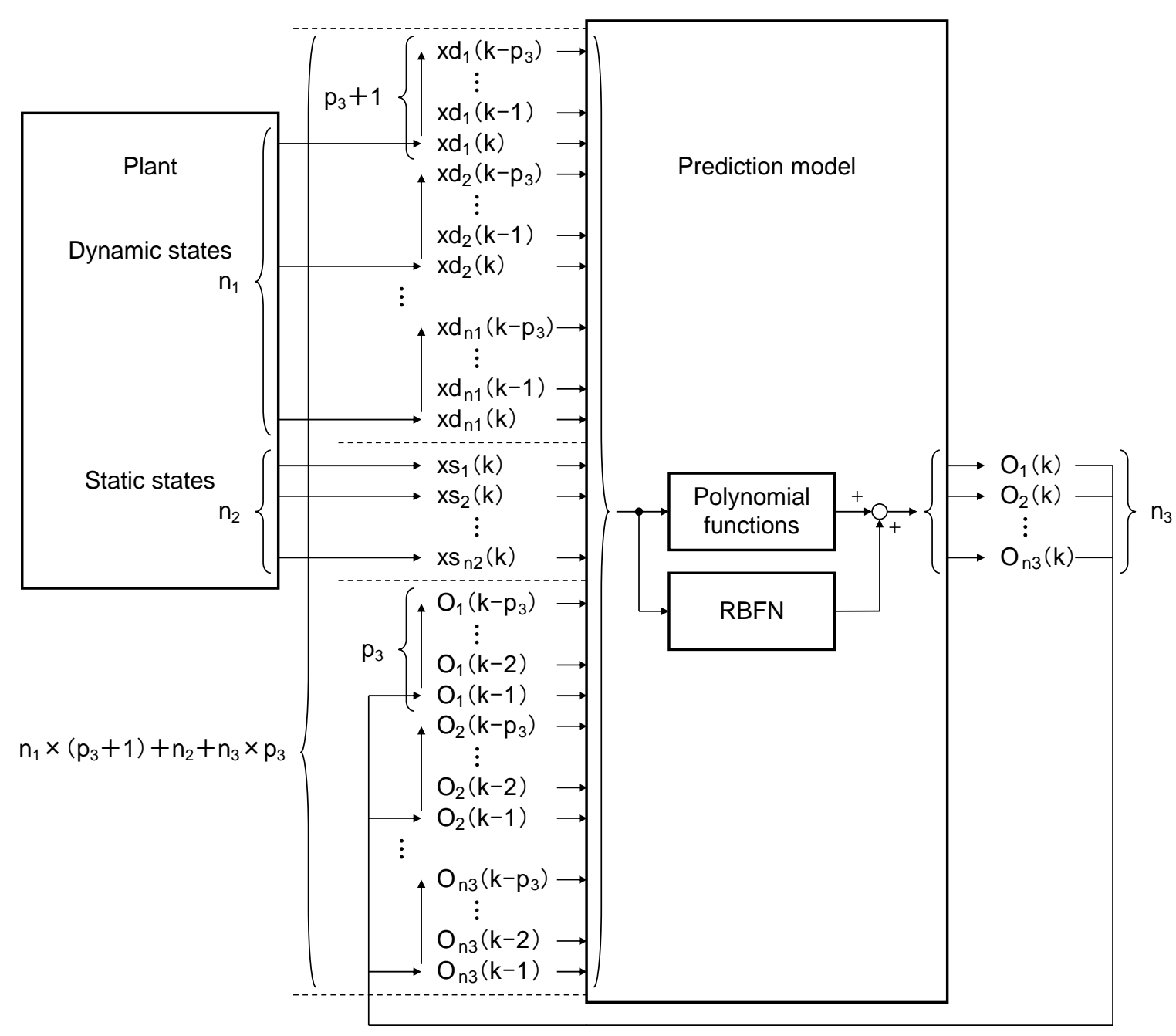

Fig. 3 Proposed RRBFN prediction model. The input values of dynamic states $x d$ and static states $x s$, respectively, are input from the plant, and the predictive output values $O$ are fed back to the input.

are datasets of RRBFN input and output values, each containing the values of quantitative variables $m=\left(n_{1}+n_{3}\right)$ $\times\left(p_{3}+1\right)+n_{2}$. To cluster observations, it is important to establish a similarity measure that joins like items. Similarity is typically determined based on the distance between observations in the $m$-dimensional variable space. We use Euclidian distance to measure the distance between two datasets (items) represented by $x$ and $y$ :

$$
d(x, y)=\sqrt{\sum_{i=1}^{m}\left(x_{i}-y_{i}\right)^{2}} .
$$

In this study, we applied agglomerative hierarchical cluster analysis with a centroid method (Everitt et al., 2011). Agglomerative hierarchical cluster analysis begins by placing each dataset (item) in a separate cluster and then combining clusters based on their distance from each other. The process continues until the desired number of clusters is formed. At each stage, the clusters joined are the closest pair. The centroid method defines the distance between two clusters as the distance between the centroids of the clusters, where each centroid is located at the average values of each variable over all members of the cluster. Finally, only the dataset closest to the centroid of each cluster is preserved as training data for the RRBFN. Thus, the number of training data becomes the same as the number of clusters. For example, we consider a simple problem with a two-dimensional variable space. As shown in Fig. 4, three clusters $-G_{1}, G_{2}$, and $G_{3}$-are enclosed with a dashed line. The $\bigcirc$ symbols indicate the centroids of the data (black points) in the clusters. The distances between the clusters are defined as $a, b$, and $c$. Distance $c$ between clusters $G_{2}$ and $G_{3}$ is the shortest. Therefore, clusters $G_{2}$ and $G_{3}$ are combined. 


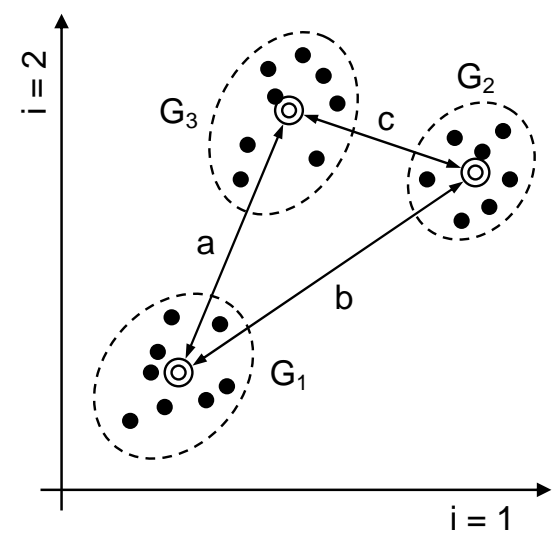

Fig. 4 Cluster analysis for a two-dimensional variable space.

\subsubsection{Verification of the proposed RRBFN prediction model}

Storing sufficient start-up data of a steam turbine before the commercial operation of a power plant begins is difficult; hence, the proposed RRBFN prediction model is premised on performing online/offline learning after commissioning operation. Therefore, the nitrogen oxide $\left(\mathrm{NO}_{\mathrm{x}}\right)$ decomposition (as shown in Fig. 5) is considered herein as substitution operation data, to verify the accuracy of the RRBFN. These operation data are suitable for the verification of the RRBFN because the $\mathrm{NO}_{\mathrm{x}}$ flow rate and the thermal stress of the stream turbine rotor have common characteristics that change in correlation with the power generation output. The proposed RRBFN prediction model was generated using the actual operation data from a thermal power plant. $\mathrm{NO}_{\mathrm{x}}$ in a combustor exhaust gas flow is decomposed by ammonia $\left(\mathrm{NH}_{3}\right)$ injection as a reducer into a catalyst chamber, and $\mathrm{NO}_{\mathrm{x}}$ is broken down into water vapor $\left(\mathrm{H}_{2} \mathrm{O}\right)$ and nitrogen $\left(\mathrm{N}_{2}\right)$. Finally, nonreacted $\mathrm{NO}_{\mathrm{x}}$ and $\mathrm{NH}_{3}$, in addition to the $\mathrm{H}_{2} \mathrm{O}$ and $\mathrm{N}_{2}$, are discharged from a chimney into the atmosphere. It is generally considered difficult to predict this chemical decomposition process with high speed and accuracy in a physical model. This reaction is nonlinear and has a long lag time. Here, the dynamic state variables are the power generation output, combustor outlet $\mathrm{NO}_{\mathrm{x}}$ flow rate, and $\mathrm{NH}_{3}$ injection flow rate, so the number of dynamic state variables is $n_{1}=3$. The $\mathrm{NO}_{\mathrm{x}}$ decomposition efficiency depends on the catalyst temperature, but the time constant of the catalyst temperature is much longer than the other state variables. Hence, the static state variable is the catalyst temperature, so the number of static state variables is $n_{2}=1$. Finally, the output variable is the chimney inlet $\mathrm{NO}_{\mathrm{x}}$ flow rate, so the number of output variables is $n_{3}=1$.

We prepared approximately 90 hours of actual plant operation data (32464 datasets) of various operational statuses, such as start-ups, shutdowns, load changes, and load rejections. One sampling period $\Delta t$ is $10 \mathrm{~s}$, and the number of past sample periods $p_{3}$ is $10(100 \mathrm{~s})$. Thus, the datasets have 45 dimensions: $m=(3+1) \times(10+1)+1=45$. Then, the number of datasets was reduced to $1 / 2$ (16232 datasets), $1 / 4$ (8116 datasets), 1/8 (4058 datasets), and 1/16 (2029 datasets) using cluster analysis. We considered the number of prediction periods $p_{2}$ to be 10 (100 s) because the delay times (e.g., transport delay, lag time, and dead time) of this process are approximately $90 \mathrm{~s}$. The RRBFN is trained using datasets of each clustering case. The RRBFN then performs a prediction calculation using test datasets of similar operation data. Figure 6 compares the predicted output value of 10 samples $(100 \mathrm{~s})$ ahead and the actual plant operation data corresponding to a load change between $100 \%$ and 50\%. Figures 6(a), 6(b), 6(c), and 6(d) illustrate the $1 / 2,1 / 4,1 / 8$, and $1 / 16$ clustering cases, respectively (made dimensionless by normalization to the value at $100 \%$ load).

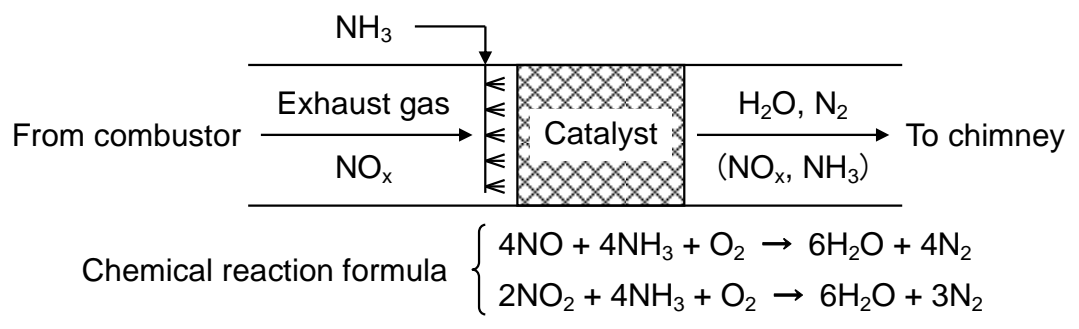

Fig. 5 Configuration of $\mathrm{NO}_{\mathrm{x}}$ decomposition in a thermal power plant. 

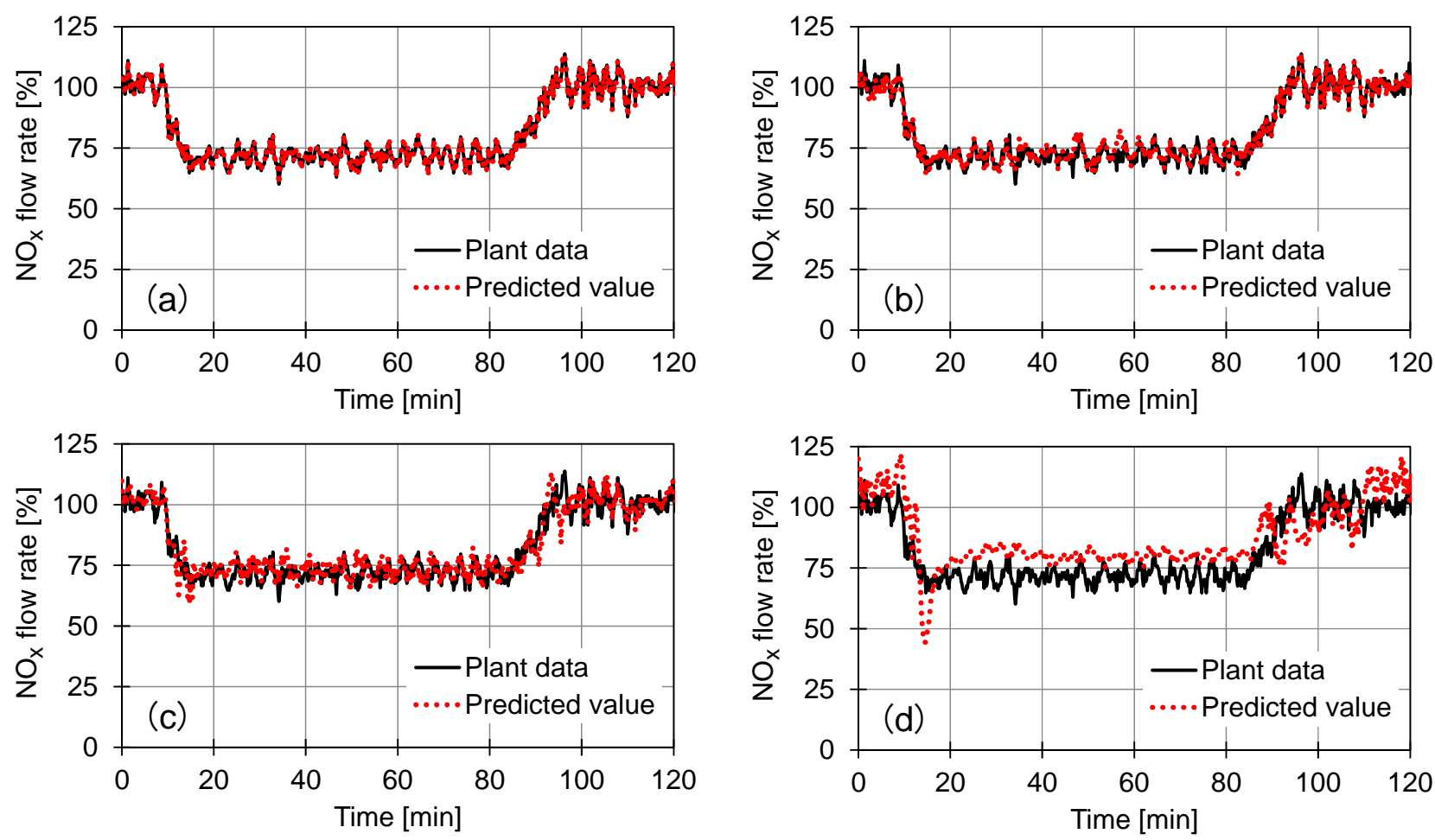

Fig. 6 Comparison of predicted and actual plant operation values of the chimney inlet $\mathrm{NO}_{\mathrm{x}}$ flow rate. (a), (b), (c), and (d) Clustering cases of $1 / 2,1 / 4,1 / 8$, and 1/16, respectively, made dimensionless by normalization to the value at $100 \%$ load.

Table 1 Computing times for training an RRBFN in each clustering case.

\begin{tabular}{|l|c|c|c|}
\hline \multicolumn{2}{|c|}{ Clustering case } & Number of datasets & Computing time \\
\hline (a) & $1 / 2$ & 16232 & $120 \mathrm{~min}$ \\
\hline (b) & $1 / 4$ & 8116 & $3 \mathrm{~min}$ \\
\hline (c) & $1 / 8$ & 4058 & $1 \mathrm{~min}$ \\
\hline (d) & $1 / 16$ & 2029 & $0.5 \mathrm{~min}$ \\
\hline
\end{tabular}

The computing times for RRBFN training in each clustering case using a personal computer (Intel ${ }^{\mathrm{TM}} \mathrm{Core}^{\mathrm{TM}} 2 \mathrm{Duo}$ processorT7500@2.20 GHz) are summarized in Table 1. The analysis results show that the prediction accuracy decreases with the number of clusters, but the computing time is considerably reduced. Of course, depending on the quality and quantity of the plant operation data, the predicted output values agree well with actual plant operation data when the number of clusters is more than $1 / 8$ (4058 datasets) in this study.

\section{Application results}

To demonstrate the effectiveness of the proposed method, we applied it to an example of start-up control of a steam power plant. Generally, optimizing the start-up of a steam turbine unit involves several conflicting objective functions, such as start-up time and the thermal stress of the steam turbine rotor. A lower rotor thermal stress further extends the service lifetime of the steam turbine. As expected, reducing the start-up time improves the load-following capability and fossil fuel economy. There exist many operational constraints for steam turbines. However, most constraints (e.g., rotor critical speed and vibration, and steam pressure and temperature) are safety-controlled to within given limits at any time. Thus, the operational constraints include only the thermal stress of the steam turbine rotor and the thermal expansion difference between a turbine rotor and labyrinth in the axial direction of the steam turbine rotor. Thermal stress is one of the objective functions, and it has an upper limit to prevent metal creep and fatigue. Thermal expansion 
increases significantly as the start-up time decreases. However, it has an upper limit determined by the speed-up and loading rates. Therefore, we considered the thermal expansion constraint indirectly by restricting the upper limit of the speed-up and loading rates in this study. In this section, we describe the configuration of the plant system, define the proposed multi-objective model predictive control problem, and present the results of a dynamic simulation study.

\subsection{Plant system configuration}

A typical steam power plant, such as that shown in Fig. 7, was considered in this study. The boiler generates steam for the steam turbine, and the steam turbine drives the generator. This steam power plant generates an output of 600 MW. A steam governing valve (GV) manipulates the steam flow rate swallowed by the steam turbine. The three sensors in Fig. 7 measure the boiler outlet steam pressure $P_{s}$, boiler outlet steam temperature $T_{s}$, and first-stage shell metal temperature of the steam turbine $T_{m}$, respectively. Generally, $T_{m}$ is regarded as equivalent to the rotor surface metal temperature of the steam turbine. The steam turbine exhaust is conducted to the boiler reheater and recovered to the condenser via a low-pressure steam turbine.

During start-up, there is a large temperature difference between the hot steam and cold steam turbine rotor, and the rotor surface is heated by the higher-temperature steam. The temperature in the outer part rises higher than that of the inner part because the rotor has a large capacitance. Thermal stress on the steam turbine rotor occurs because of this temperature difference. It is difficult to measure the actual rotor thermal stress directly because the turbine rotor rotates at high speed through high-temperature steam. Therefore, the thermal stress of the steam turbine rotor is obtained by the temperature distribution, which is calculated by a dynamic model of thermal conduction that divides the rotor into several vertical cylinders. Rotor thermal stress is described in Eqs. (25) and (26); it can be calculated by estimating the temperature distribution inside the rotor from Eq. (25), using the time-series behavior of the measured $T_{m}$ as the boundary condition. Then, the values of the inner bore and outer surface of the rotor thermal stress (where thermal stress is concentrated) are calculated using Eq. (26).

$$
\begin{aligned}
& \frac{\partial \theta}{\partial \tau}=\frac{\lambda_{m}}{c_{m} \rho_{m}}\left(\frac{\partial^{2} \theta}{\partial r^{2}}+\frac{1}{r} \frac{\partial \theta}{\partial r}\right), \\
& \sigma_{z z}=\frac{E_{m} \alpha_{m}}{1-v_{m}}\left(\frac{2}{R_{o}^{2}-R_{i}^{2}} \int_{R_{i}}^{R_{o}} \theta r \mathrm{~d} r-\theta\right),
\end{aligned}
$$

where $c_{m}$ represents the specific heat; $E_{m}$, Young's modulus; $r$, the radial axis; $R_{i}$ and $R_{o}$, the inner and outer radii, respectively; $\alpha_{m}$, the coefficient of linear expansion; $\theta$, the rotor temperature; $\lambda_{m}$, the thermal conductivity; $v_{m}$, Poisson's ratio; $\rho_{m}$, the density; $\sigma_{z z}$, the thermal stress; and $\tau$, the time.

The steam turbine speed/load controller modulates the manipulative variable (MV) for the GV to adjust the process

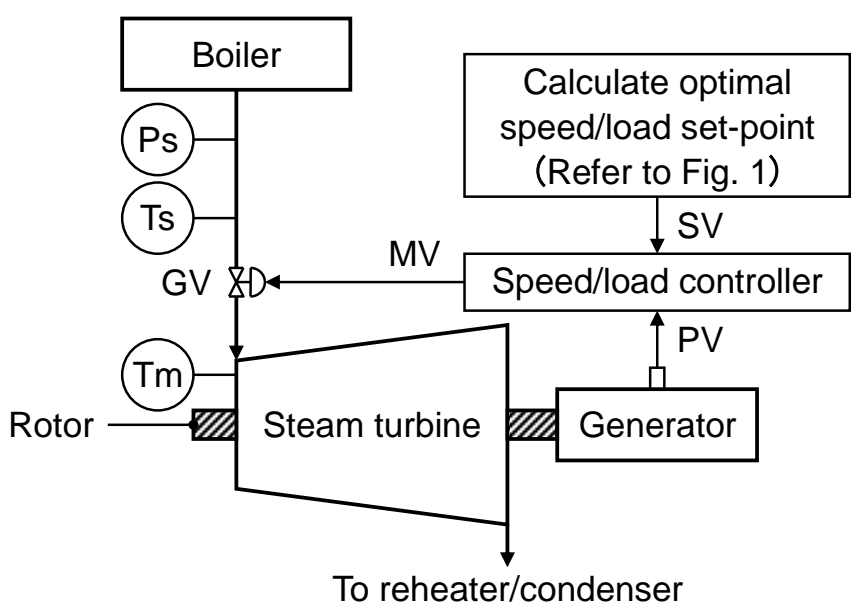

Fig. 7 Configuration of a typical steam power plant system. The speed and load set-points are derived from the proposed plant control system in Fig. 1. 
variable (PV) of the actual generator speed/load to its set variable (SV) from the multi-objective model predictive control system shown in Fig. 1.

\subsection{Problem definitions for the proposed multi-objective model predictive control}

As the parameter times of model predictive control in Fig. 2, we set the sampling period (optimization cycle) $\Delta t$ to $1 \mathrm{~min}$, prediction period $p_{1}$ to $20 \mathrm{~min}$ ( 20 samples), control period $p_{2}$ to $4 \mathrm{~min}$ ( 4 samples), and past sample period $p_{3}$ to $5 \mathrm{~min}$ ( 5 samples). These parameters were determined by adjusting each of them to a value that would enable completion of the optimization calculations within one sampling period $\Delta t$ while confirming the validity of the control response. In this section, we discuss the formulation of this optimizer and describe the input and output variables of the RRBFN prediction model.

\subsubsection{Optimizer \\ Design variables}

The speed-up and loading rates of steam turbine $\Delta u$ during control period $p_{2}$ are treated as design variables. Since the steam turbine generator automatically synchronizes with the electrical grid to raise the load after reaching the rated speed, the number of design variables is the same as the number of samples in control period $p_{2}$. The speed and load of steam turbine $u$ can be obtained by integrating as follows:

$$
\Delta \boldsymbol{u}=\left[\begin{array}{c}
\Delta u(t) \\
\Delta u(t+1) \\
\vdots \\
\Delta u\left(t+p_{2}-1\right)
\end{array}\right], \quad \boldsymbol{u}=\left[\begin{array}{c}
u(t+1)=u(t)+\Delta u(t) \\
u(t+2)=u(t+1)+\Delta u(t+1) \\
\vdots \\
u\left(t+p_{2}\right)=u\left(t+p_{2}-1\right)+\Delta u\left(t+p_{2}-1\right)
\end{array}\right],
$$

where $t$ represents the present time. The speed/load of the steam turbine at the present time $u(t)$ is given by the actual observed value $u(t)=u_{o}$.

\section{Objective functions}

The start-up time and rotor thermal stress of the steam turbine are treated as objective functions. The start-up time $f_{1}$ begins at the rolling-off of the steam turbine, ends at the realization of plant base-load operation, and corresponds to terminal time $T$ in the mathematical model of Eq. (1). If the steam turbine load does not reach the base-load operation during prediction period $p_{1}$, the steam turbine load is extrapolated to estimate $f_{1}$. Otherwise, $f_{1}$ is the time taken to reach the base-load operation, which is the terminal time $T$. Therefore, $f_{1}$ during $p_{1}$ is minimized as follows:

$$
\min f_{1}=\left\{\begin{array}{l}
t+\left[p_{1}+\frac{u_{S, \max }-u\left(t+p_{1}\right)}{\Delta u_{S}\left(t+p_{1}\right)}+\frac{u_{L, \max }}{\Delta u_{L, \text { table }}}\right] \cdot \Delta t, \quad \text { if } u\left(t+p_{1}\right)<u_{S, \max } \text { (before loading), } \\
t+\left[p_{1}+\frac{u_{L, \max }-u\left(t+p_{1}\right)}{\Delta u_{L}\left(t+p_{1}\right)}\right] \cdot \Delta t, \quad \text { if } u_{S, \max }=u\left(t+p_{1}\right)<u_{L, \text { max }} \text { (during loading), } \\
T, \quad \text { otherwise },
\end{array}\right.
$$

where $u_{S, \max }$ represents the steam turbine speed at the rated speed; $\Delta u_{S}\left(t+p_{1}\right)$, the predicted value of the steam turbine speed-up rate at the last time in prediction period $t+p_{1} ; u_{L, \text { max }}$, the steam turbine load in base-load (target load) operation; and $\Delta u_{L}\left(t+p_{1}\right)$, the predicted value of the steam turbine loading rate at the last time in prediction period $t+p_{1}$. For convenience, the loading rate $\Delta u_{L \text {, table }}$ is assumed by linearly interpolating from the speed-up rate $\Delta u_{S}\left(t+p_{1}\right)$ using Table $\mathrm{A}$ in the Appendices.

The thermal stress of steam turbine rotor $f_{2}$ is the maximum value during start-up. It is used to calculate the values of the rotor bore and rotor surface at which the thermal stress is concentrated and to set their maxima. Therefore, $f_{2}$ during $p_{1}$ can be minimized as follow:

$$
\min f_{2}=\max \left[\sigma_{\max }, \hat{\sigma}_{b}(k), \hat{\sigma}_{s}(k)\right], \quad k=t+1, t+2, \cdots, t+p_{1},
$$


where $\hat{\sigma}_{b}$ and $\hat{\sigma}_{s}$ represent the predicted values of the rotor bore and rotor surface thermal stresses, respectively, of the steam turbine during $p_{1}$. The maximum thermal stress of the steam turbine rotor during the past time $\sigma_{\max }$ is given by actual observed values as follows:

$$
\sigma_{\max }=\max \left[\sigma_{b}(k), \sigma_{s}(k)\right], \quad k=0,1, \cdots, t,
$$

where $\sigma_{b}$ and $\sigma_{s}$ represent observed values of the rotor bore and rotor surface thermal stresses, respectively, and are calculated using Eqs. (25) and (26) based on actual observed first-stage shell metal temperature $T_{m}$.

\section{Constraining conditions}

Due to the thermal expansion discussed at the beginning of this section, limiting values are added to the speed-up and loading rates of the steam turbine, which are the design variables in Eq. (27). Since the upper and lower bounds of the design variables can be limited by the ranges of the genetic operations described in Section 3.2, they need not be treated as penalty functions.

Before synchronizing the steam turbine generator to the electrical grid, the speed-up rate $\Delta u$ is determined using Eq. (31). Here, between turbine speeds of $30 \%$ and $90 \%$, the lower limit is set to avoid critical speeds.

$$
\text { Speed-up rate }\left\{\begin{array}{l}
0 \% / \mathrm{min} \leq \Delta u \leq 10 \% / \mathrm{min}, \quad \text { if } 0 \% \leq u<30 \% \\
3.333 \% / \mathrm{min} \leq \Delta u \leq 10 \% / \mathrm{min}, \quad \text { if } 30 \% \leq u \leq 90 \% \\
0 \% / \mathrm{min} \leq \Delta u \leq 10 \% / \mathrm{min}, \quad \text { if } 90 \%<u \leq 100 \%
\end{array}\right.
$$

The speed-up rate of the steam turbine $\Delta u$ from time $t+p_{2}$ to $t+p_{1}-1$ is fixed at the last value in the control period $\Delta u\left(t+p_{2}-1\right)$. The steam turbine speed $u$ is obtained by integrating as follows:

$$
\left\{\begin{array}{l}
\Delta u(k)=\Delta u\left(t+p_{2}-1\right), \\
u(k+1)=u(k)+\Delta u(k), \quad k=t+p_{2}, t+p_{2}+1, \cdots, t+p_{1}-1 .
\end{array}\right.
$$

The predicted steam turbine speed-up rate at the last time in the prediction period $\Delta u_{S}\left(t+p_{1}\right)$ in Eq. (28) is given by

$$
\Delta u_{S}\left(t+p_{1}\right)=\max \left[\Delta u_{S, \min }, \Delta u\left(t+p_{1}-1\right)\right] .
$$

For convenience, we set lower limit $\Delta u_{S \text {, min }}$ to $3.333 \% / \mathrm{min}$.

When the steam turbine generator is synchronized to the electrical grid, the loading rate $\Delta u$ is given by Eq. (34). Here, between turbine loads of $0 \%$ and $5 \%$, the lower limit is set to ensure power stabilization at the initial load.

$$
\text { Loading rate }\left\{\begin{array}{l}
0.5 \% / \mathrm{min} \leq \Delta u \leq 10 \% / \mathrm{min}, \quad \text { if } 0 \% \leq u \leq 5 \%, \\
0 \% / \mathrm{min} \leq \Delta u \leq 10 \% / \mathrm{min}, \quad \text { if } 5 \%<u \leq 100 \% .
\end{array}\right.
$$

The loading rate of the steam turbine $\Delta u$ from time $t+p_{2}$ to $t+p_{1}-1$ is fixed at the last value in the control period $\Delta u\left(t+p_{2}-1\right)$. The steam turbine load $u$ is obtained by integrating as follows:

$$
\left\{\begin{array}{l}
\Delta u(k)=\Delta u\left(t+p_{2}-1\right), \\
u(k+1)=u(k)+\Delta u(k), \quad k=t+p_{2}, t+p_{2}+1, \cdots, t+p_{1}-1
\end{array}\right.
$$

The predicted steam turbine loading rate at the last time in the prediction period $\Delta u_{L}\left(t+p_{1}\right)$ in Eq. (28) is given by

$$
\Delta u_{L}\left(t+p_{1}\right)=\max \left[\Delta u_{L, \min }, \Delta u\left(t+p_{1}-1\right)\right] .
$$

For convenience, we set lower limit $\Delta u_{L, \min }$ to $0.5 \% / \mathrm{min}$. However, when $u$ is higher than $50 \%$, the boiler outlet steam temperature $T_{s}$ is statically determinate (refer to Table A in the Appendices) and the thermal stress of the steam turbine rotor is reduced, so $\Delta u_{L, \min }$ is switched to $10 \% / \mathrm{min}$. 
The thermal stress of steam turbine rotor $f_{2}$ has the following upper limit:

$$
g=f_{2}-\sigma_{U} \leq 0
$$

where $\sigma_{U}$ is the upper limit of the thermal stress of the steam turbine rotor. The constraining condition of Eq. (37) is considered in the penalty function of Eq. (11).

\subsubsection{Prediction model}

Since the mechanical structure of the steam turbine is complex, it is difficult to predict the thermal stress of the steam turbine rotor accurately with a short computing time using only a physical model. Therefore, we will predict the behavior of the first-stage shell metal temperature of the steam turbine $T_{m}$ in the future using the RRBFN proposed in Section 3.3.1. The thermal stress of the steam turbine rotor, which is one of the objective functions, is predicted by solving the physical model of Eqs. (25) and (26) with $T_{m}$ as a boundary condition. That is, by using this gray-box model that combines the physical (white-box) and RRBFN (black-box) models, the computing time and accuracy can be balanced. The analytical method for implementing Eqs. (25) and (26) on the controller is well known from conventional research (Matsumoto et al., 1982; Nakai et al., 1996), so its description is omitted here. $T_{m}$ is strongly correlated with boiler outlet steam pressure $P_{s}$, boiler outlet steam temperature $T_{s}$, and steam turbine speed and load $u$. The RRBFN input and output variables during prediction period $p_{1}$ are summarized below:

Input variables $\left\{\begin{array}{l}P_{s}\left(k-p_{3}\right), \cdots, P_{s}(k-1), P_{s}(k), \\ T_{s}\left(k-p_{3}\right), \cdots, T_{s}(k-1), T_{s}(k), \\ u\left(k-p_{3}\right), \cdots, u(k-1), u(k), \\ T_{m}\left(k-p_{3}\right), \cdots, T_{m}(k-2), T_{m}(k-1), \quad k=t+1, t+2, \cdots, t+p_{1},\end{array}\right.$

Output variable $T_{m}(k), \quad k=t+1, t+2, \cdots, t+p_{1}$.

Here, $T_{m}$, which is one of the input variables, is the feedback value of the predicted output variable. The steam turbine speed and load $u$ in future times $\left[t+1, t+p_{1}\right]$ are given by the optimizer described above. For convenience, the boiler outlet steam pressure $P_{S}$ and temperature $T_{s}$ in $\left[t+1, t+p_{1}\right]$ are assumed by linearly interpolating from the steam turbine speed and load $u$ using Table B in the Appendices. Since $P_{s}$ and $T_{s}$ are controlled sufficiently faster than $T_{m}$ responds; even if they are given by a static function, few problems occur. However, the optimal controls and prediction methods of $P_{s}$ and $T_{s}$ are left for future research. Input variables $\left(P_{s}, T_{s}, u\right.$, and $\left.T_{m}\right)$ from past times $\left[t-p_{3}+1, t\right]$ are determined based on actual observed values.

\subsection{Results of the dynamic simulation study}

Prior to application to a real power plant, we verified the proposed multi-objective model predictive control method by performing dynamic simulation studies of a $600 \mathrm{MW}$ steam power plant. The dynamic simulation models were verified using actual plant data (Shirakawa et al., 2005; Shirakawa, 2006). The simulation was executed under warm start-up conditions in which the initial metal temperature of the steam turbine rotors was $170^{\circ} \mathrm{C}$.

The dynamic simulation was executed for each aspiration level with the following procedure:

1. Initial training of the RRBFN prediction model was performed using the simulation results from two cases - the fastest and slowest start-up operations of the plant (corresponding to points A and B, respectively, in Fig. 8(a)). In these cases, the upper limit of the rotor thermal stress can be neglected.

2. The plant start-up simulation was executed three times during online learning of the RRBFN prediction model.

Figures 9, 10, and 11 show the plant start-up simulation results. The steam turbine speed (broken line) and load (solid line) in Figs. 9(a), 10(a), and 11(a) are depicted as dimensionless using the rated speed and base-load operation values, respectively. The optimization calculations were executed after rub-check holding at a speed of $13.333 \%$. The rotor bore (broken line) and rotor surface (solid line) thermal stresses of the steam turbine in Figs. 9(b), 10(b), and 11(b) are shown as dimensionless by normalization to the upper limits. Figure 8 shows the result of the multi-objective optimization process as a relation between start-up time $f_{1}$ and the maximum value of the normalized rotor thermal stresses during start-up $f_{2}$. The ideal point (the symbol $*$ in Fig. 8$)$ is set to $\left(f_{1}^{*}, f_{2}^{*}\right)=(0$ min, $0 \%)$. The black circle 
points in Fig. 8(a) are the Pareto-optimal solutions obtained by changing the upper limit of the rotor thermal stress. That is, these solutions were obtained by partially modifying the proposed method to time-optimal model predictive control based on a single objective.

First, the results of time-optimal model predictive control with rotor thermal stress as a constraining condition are shown in Fig. 9. The qualitative trend of these results is very similar to that obtained by applying the previous steam turbine start-up of model predictive control (Nakai et al., 1996). Specifically, the start-up time required to reach the base-load operation is shortened by increasing the steam turbine speed and load while keeping the rotor thermal stresses at $100 \%$ of the upper limit. This result corresponds to point $\mathrm{C}$ in Fig. 8(a). That is, $\left(f_{1}, f_{2}\right)=(68 \mathrm{~min}, 100 \%)$.

Next, we discuss the results of the proposed multi-objective model predictive control. Suppose that the user wants to decrease $f_{2}$ (thermal stress). Since above-mentioned solution (point C in Fig. 8(a)) is already a Pareto-optimal solution, it is impossible to improve both criteria. Therefore, suppose that the user agrees to relax $f_{1}$ (start-up time) and let the first aspiration level be $\left(\overline{f_{1}}, \overline{f_{2}}\right)=(100 \mathrm{~min}, 100 \%)$ (the symbol $\square$ in Fig. 8(a)). Then, the first optimal solution is $\left(f_{1}, f_{2}\right)=(86 \mathrm{~min}, 90 \%)$ (the symbol $\square$ in Fig. 8(a)); here, both criteria are improved from the first aspiration level. Moreover, suppose that the user agrees to relax $f_{1}$ further and let the second aspiration level be $\left(\overline{f_{1}}, \overline{f_{2}}\right)=(120 \mathrm{~min}, 90 \%)$ (the symbol $\Delta$ in Fig. 8(a)). Then, the second optimal solution is $\left(f_{1}, f_{2}\right)=(96 \mathrm{~min}, 77 \%)$ (the symbol $\Delta$ in Fig. 8(a)); here, both criteria are improved from the second aspiration level. However, the second
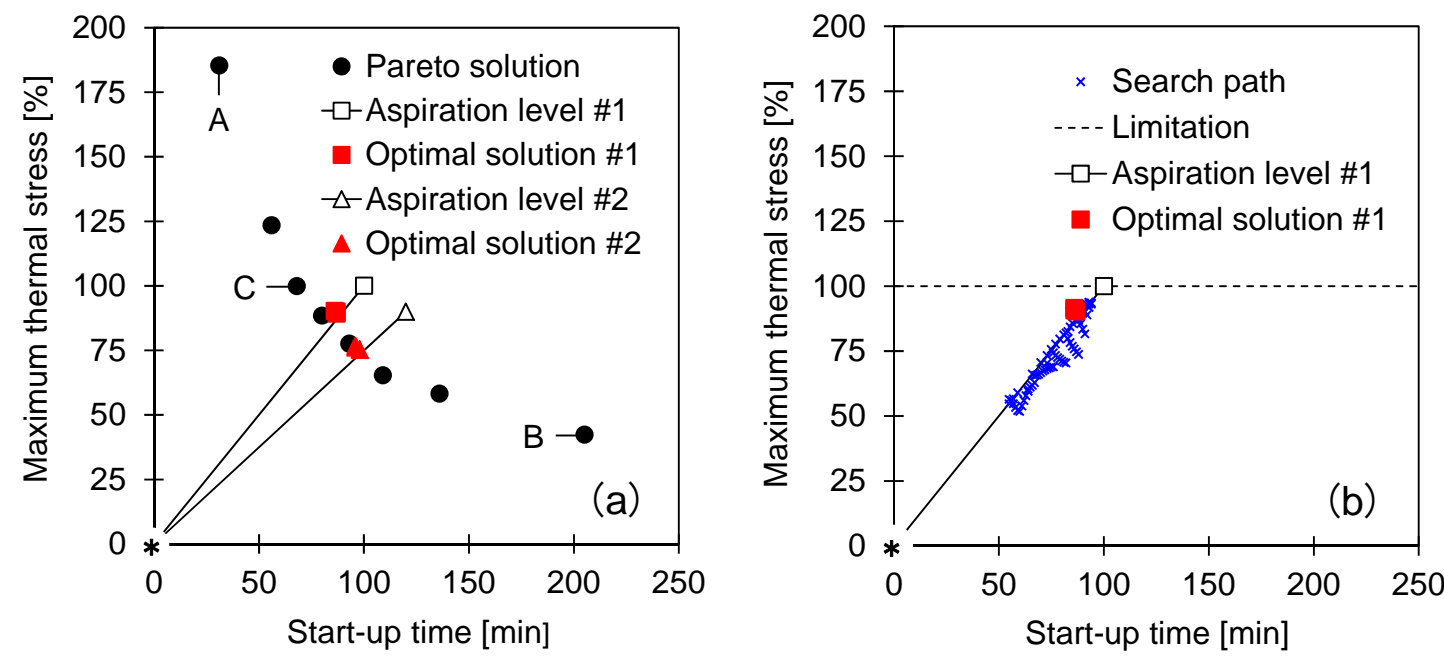

Fig. 8 Results of multi-objective optimization. (a) Aspiration levels and comparison of the obtained optimal solutions to the Pareto-optimal solutions. (b) Search path history for the first optimal solution.
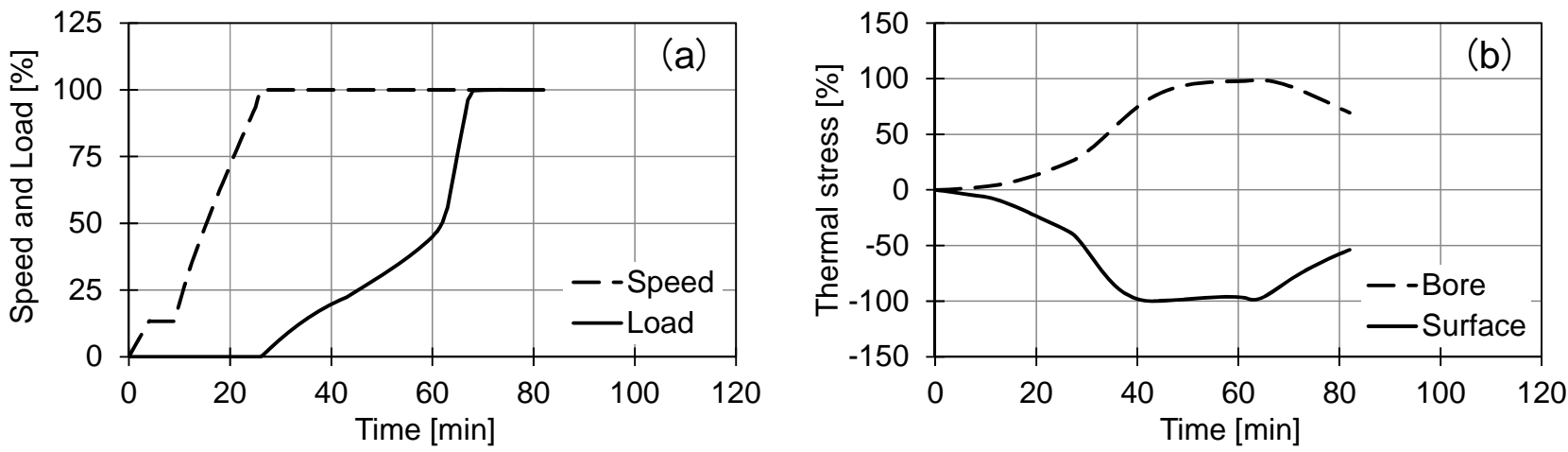

Fig. 9 Dynamic simulation results obtained for time-optimal model predictive control corresponding to point $\mathrm{C}$ in Fig. 8(a). (a) Steam turbine speed and load in single-objective control. (b) Rotor bore and rotor surface thermal stresses. 

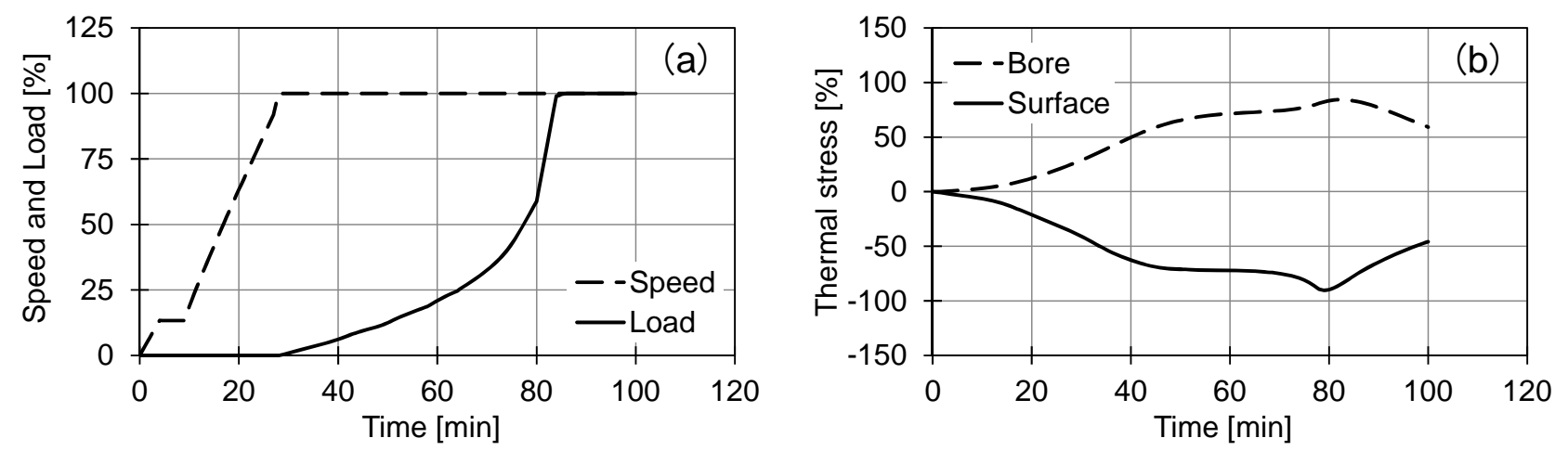

Fig. 10 Dynamic simulation results obtained for the first aspiration level. (a) Steam turbine speed and load with the proposed multi-objective control. (b) Rotor bore and rotor surface thermal stresses.
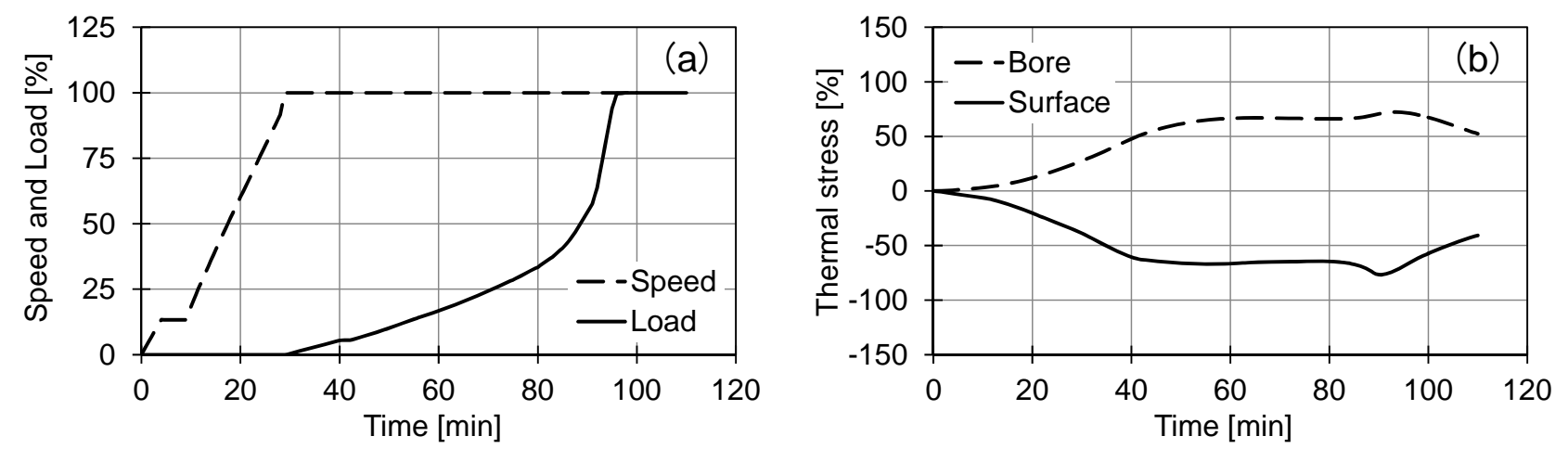

Fig. 11 Dynamic simulation results obtained for the second aspiration level. (a) Steam turbine speed and load with the proposed multi-objective control. (b) Rotor bore and rotor surface thermal stresses.

optimal solution shows that $f_{2}$ improves, but $f_{1}$ deteriorates compared to the first optimal solution, because there is a trade-off between $f_{1}$ and $f_{2}$. The first optimal solutions in Fig. 8(a) all plot the results of three plant start-up simulations, but they are almost the same. The deviations in these solutions are only within 0.667 min for $f_{1}$ and $0.619 \%$ for $f_{2}$. Likewise, the second optimal solutions in Fig. 8(a) all plot the results of three plant start-up simulations, but they are almost the same. The deviations in these solutions are only within 1.333 min for $f_{1}$ and $0.774 \%$ for $f_{2}$. Since the optimal solutions are reproducible, the learning of the RRBFN prediction model is satisfactory. Although some gap results from the influence of the prediction error of the start-up time in Eq. (28), all these solutions obtaining multi-objective control substantially agree with the Pareto-optimal solutions obtaining single-objective control closest to each aspiration level. This agreement demonstrates the validity of the optimal solutions obtained using the proposed method. The control responses of the first optimal solution in Fig. 10 are the results of the third start-up simulation. This multi-objective control satisfies the operating constraints in all periods; the rotor thermal stresses in Fig. 10(b) are particularly reduced from the time-optimal control results in Fig. 9(b). Note that a peak rotor thermal stress occurs around $80 \mathrm{~min}$. Figure 8 (b) plots the search path history for this optimal control input at each sampling time. In the time-optimal control, the rotor thermal stress is limited to a constant upper limit along the broken line in Fig. 8(b). In contrast, in the proposed multi-objective control, the objective functions are optimized along the solid line in Fig. 8(b) (the line connecting the aspiration level and the ideal point), so that the rotor thermal stress fluctuates near the Pareto-optimal solution. However, this peak can be made smaller by adjusting the prediction period $p_{1}$ and control period $p_{2}$. The control responses of the second optimal solution in Fig. 11 are the results of the third start-up simulation. The control responses of the second optimal solution show that the start-up time is longer than it is for the first optimal solution and that the rotor thermal stresses are reduced. In this way, the proposed method has features that enable flexible online control based on multi-objective evaluation according to aspiration levels. 


\subsection{Computing time}

We intend to implement the proposed multi-objective model predictive control method in an actual plant controller in future research. Here, we discuss the computing time of the proposed method, which is important for practical use. The GA parameters greatly affect the computing time. In Section 3.2, we used 20 individuals and set the maximum number of iterations to 50 generations. We confirmed that the solutions converged before 50 generations occurred. We executed start-up simulations using a personal computer (Intel ${ }^{\mathrm{TM}}$ Core $^{\mathrm{TM}}$ i7-3770S CPU @ 3.10 GHz). In this case, it took approximately $33 \mathrm{~s}$ (searching using the GA for approximately $9 \mathrm{~s}$, learning and predicting the metal temperature for the RRBFN for approximately $6 \mathrm{~s}$, and calculating the rotor thermal stress based on the physical model for approximately $18 \mathrm{~s}$ ) to obtain the optimal control input per sampling period. Any case can be processed within the $60 \mathrm{~s}$ sampling period. This time is sufficiently short for practical control problems such as steam turbine start-up.

\section{Conclusions}

We proposed herein a novel intelligent multi-objective model predictive control method, in which an RRBFN was applied for model prediction, and the satisficing trade-off method was used for multi-objective optimization. With this control method,

1. several conflicting control objectives can be optimized in online control based on multi-objective evaluation through human-computer interaction;

2. optimal and flexible plant control can be performed within a restrained practical computing time for real-time applications and with acceptable control quality using online adaptive model prediction.

In addition, the proposed method maintained the framework of the previously reported human-computer interaction. Therefore, after optimizing the control schedule offline using a previous optimization system (Shirakawa et al., 2007), the proposed method can be used for online optimal control.

The proposed method worked well throughout our experiments. Our main results are as follows:

1. The accuracy of the proposed RRBFN prediction model was verified using the actual operation data of a $\mathrm{NO}_{\mathrm{x}}$ decomposition process in a thermal power plant.

2. The RRBFN training data size can be reduced using a cluster analysis, which was important for maintaining predictive accuracy and shortening the learning time of the RRBFN prediction model.

3. The proposed multi-objective model predictive control method was applied to steam turbine start-up control. Although some gap resulted from the influence of the prediction error of the start-up time, the solutions obtained through dynamic simulations substantially agreed with the Pareto-optimal solutions, which were given by single objectives with constraints. However, the multi-objective control responses partially differed from the single-objective results with constraints in its search path history.

4. Our application results indicated that the computing time of the proposed method was practical for implementation in a controller in the future research.

This study demonstrated the verification results of the proposed control method through dynamic simulations. The future work will field-test the proposed control method on a real power plant and add other operational constraints (e.g., $\mathrm{NO}_{\mathrm{x}}$ emission from the plant described in Section 3.3.3, etc.)

\section{Acknowledgements}

We would like to thank Professor Emeritus Hirotaka Nakayama of Konan University, whose comments and suggestions were of inestimable value to our study.

\section{Appendices}

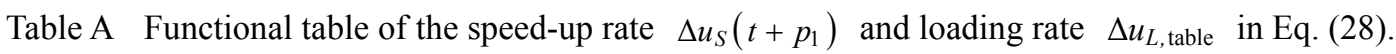

\begin{tabular}{c|c|c|c|c|c|}
\hline Speed-up rate & {$[\% / \mathrm{min}]$} & 0 & 3.333 & 5 & 10 \\
\hline Loading rate & {$[\% / \mathrm{min}]$} & 0 & 0.5 & 3 & 6 \\
\hline
\end{tabular}


Table B Functional table of the steam turbine speed and load $u$, and the boiler outlet steam pressure $P_{s}$ and temperature $T_{s}$.

\begin{tabular}{|c|c|c|c|c|c|c|c|c|}
\hline & & \multicolumn{2}{|c|}{ Speed } & \multicolumn{5}{c}{ Load } \\
\hline $\mathrm{u}$ & {$[\%]$} & 0 & 100 & 0 & 25 & 50 & 99.75 & 100 \\
\hline $\mathrm{P}_{\mathrm{S}}$ & {$[\mathrm{MPa}]$} & 8.46 & 8.46 & 8.46 & 8.46 & 9.34 & 16.66 & 16.66 \\
\hline $\mathrm{T}_{\mathrm{S}}$ & {$\left[{ }^{\circ} \mathrm{C}\right]$} & 350 & 410 & 410 & 474 & 538 & 538 & 538 \\
\hline
\end{tabular}

\section{References}

Bemporad, A. and Muñoz de la Peña, D., Multiobjective model predictive control, Automatica, Vol.45, Issue 12 (2009), pp.2823-2830.

Biyik, E., Long, C. E., D’Amato, F. J., Di Palma, S. and Baker, D. A., Enhanced operational flexibility of combined cycle through advanced controls, 55th ISA Power Industry Division Symposium 2012, Vol.492, (2012), POWID 2012, pp.204-213, Austin, TX, United States.

Cressie, N., Spatial prediction and ordinary Kriging, Mathematical Geology, Vol.20, No.4 (1988), pp.405-421.

D'Amato, F. J., Industrial application of a model predictive control solution for power plant startups, Proceedings of the IEEE International Conference on Control Applications 2006, (2006), CACSD-CCA-ISIC 2006, pp.243-248, Munich, Germany.

Eshelman, L. J. and Schaffer, J. D., Real-coded genetic algorithms and interval-schemata, Foundations of Genetic Algorithms 2, (1993), pp.187-202, Morgan Kaufmann Publishers, San Matero.

Everitt, B. S., Landau, S., Leese, M. and Stahl, D., Cluster Analysis, 5th Edition, (2011), John Wiley \& Sons, Inc., New York.

He, D., Wang, L. and Sun, J., On stability of multiobjective NMPC with objective prioritization, Automatica, Vol.57, (2015), pp.189-198.

Maciejowski, J. M., Predictive Control with Constrains, (2002), Pearson Education Limited, Prentice Hall, London.

Matsumoto, H., Sato, Y., Kato, F., Eki, Y., Hisano, K. and Fukushima, K., Turbine control system based on prediction of rotor thermal stress, IEEE Transactions on Power Apparatus and System, Vol.101, Issue 8 (1982), pp.25042512.

Montgomery, D. C., Design and Analysis of Experiments, 9th Edition, (2017), John Wiley \& Sons, Inc., New York.

Myers, R. H. and Montgomery, D. C., Response Surface Methodology: Process and Product Optimization Using Designed Experiments, 4th Edition, (2016), John Wiley \& Sons, Inc., New York.

Nakai, A., Nakamoto, M., Kakehi, A. and Hayashi, S., Turbine start-up algorithm based on prediction of rotor thermal stress, IFAC Proceeding Volumes, Vol.29, No.1 (1996), 13th World Congress of IFAC, pp.6867-6872, San Francisco, USA.

Nakayama, H., Aspiration level approach to interactive multi-objective programming and its applications, In: Advances in Multicriteria Analysis (Pardalos, P. M., Siskos, Y. and Zopounidis, C., Eds.), (1995), pp.147-174, Kluwer Academic Publishers, The Netherlands.

Nakayama, H., Arakawa, M. and Sasaki, R., Simulation-based optimization using computational intelligence, Optimization and Engineering, Vol.3 (2002), pp.201-214, Kluwer Academic Publishers, The Netherlands.

Nakayama, H., Yun, Y. and Yoon, M., Sequential approximate optimization, In: Sequential Approximate Multiobjective Optimization Using Computational Intelligence (Yoon, M., Yun, Y. and Nakayama, H., Eds.), (2009), pp.113-149, Springer-Verlag, Berlin, Heidelberg.

Núñez, A., Cortés, C. E., Sáez, D., Schutter, B. D. and Gendreau, M., Multiobjective model predictive control for dynamic pickup and delivery problems, Control Engineering Practice, Vol.32, (2014), pp.73-86.

Papadrakakis, M., Lagaros, N. D. and Tsompanakis, Y., Structural optimization using evolution strategies and neural networks, Computer Methods in Applied Mechanics and Engineering, Vol.156, Issue 1-4 (1998), pp.309-333.

Rojas, C. A., Rodriguez, J., Villarroel, F., Espinoza, J. R., Silva, C. A. and Trincado, M., Predictive torque and flux control without weighting factors, IEEE Transactions on Industrial Electronics, Vol.60, Issue 2 (2013), Article number 6239590, pp.281-690. 
Schuermann, N., Idelchi, A. and Yang, S., Model predictive control for increased steam turbine operational flexibility, 59th ISA Power Industry Division Symposium 2016, (2016), POWID 2016, pp.16-28, Charlotte, NC, United States.

Shirakawa, M., Nakamoto, M. and Hosaka, S., Dynamic simulation and optimization of start-up processes in combined cycle power plants, JSME International Journal, Series B, Vol.48, No.1 (2005), pp.122-128.

Shirakawa, M., Development of a thermal power plant simulation tool based on object orientation, Proceedings of the IMechE, Part A: Journal of Power and Energy, Vol.220, No.6 (2006), pp.569-579.

Shirakawa, M., Arakawa, M. and Nakayama, H., Intelligent start-up schedule optimization system for a thermal power plant, JSME Technical Journal, Journal of Advanced Mechanical Design, Systems, and Manufacturing, Vol.1, No.5 (2007), pp.690-705.

Tica, A., Gueguen, H., Dumur, D., Faille, D. and Davelaar, F., Hierarchical nonlinear model predictive control for combined cycle start-up optimization, Proceedings of the IEEE Conference on Decision and Control 2012, Article number 6425843, (2012), CDC 2012, pp.2593-2598, Maui, HI, United States.

Wang, G. G. and Shan, S., Review of metamodeling techniques in support of engineering design optimization, Transactions of the ASME, Journal of Mechanical Design, Vol.129, No.4 (2006), pp.370-380.

Yun, Y. and Nakayama, H., Ensembled support vector machines for meta-modeling, In: Knowledge and Systems Sciences (Chen, J., Nakamori, Y., Yue, W. and Tang, X., Eds.), Communications in Computer and Information Science, Vol.660, (2016), pp.203-212, Springer, Singapore.

Zavala, V. M. and Flores-Tlacuahuac, A., Stability of multiobjective predictive control: A utopia-tracking approach, Automatica, Vol.48, Issue 10 (2012), pp.2627-2632. 